\title{
On the Closedness of the Linear Image of a Closed Convex Cone
}

\author{
Gábor Pataki \\ Department of Statistics and Operations Research \\ University of North Carolina, CB \#3260, Chapel Hill, NC 27599 \\ email: gabor@unc.edu http://www.unc.edu/〜pataki
}

When is the linear image of a closed convex cone closed? We present very simple, and intuitive necessary conditions, which

- unify, and generalize seemingly disparate, classical sufficient conditions: polyhedrality of the cone, and "Slater" type conditions;

- are necessary and sufficient, when the dual cone belongs to a class, that we call nice cones. Nice cones subsume all cones amenable to treatment by efficient optimization algorithms: for instance, polyhedral, semidefinite, and $p$-cones.

- provide similarly attractive conditions for an equivalent problem: the closedness of the sum of two closed convex cones.

Key words: closedness; linear image; closed convex cone; sum of closed convex cones; duality; common root of Slater's condition and polyhedrality

MSC2000 Subject Classification: Primary: 90C46, 49N15; Secondary: 52A40, 52A99

OR/MS subject classification: Primary: Convexity; Secondary: Programming/Nonlinear/Theory

History: Received: Xxxx xx, xxxx; revised: Yyyyyy yy, ууyy and Zzzzzz zz, zzzz.

1. Introduction. One of the most fundamental questions of convex analysis is also the simplest:

When is the linear image of a closed convex set closed?

Essential applications include: finding out, when the sum and convolution of closed convex functions is closed; and uniform duality in conic linear systems. For the first, see for instance Chapter 9 in Rockafellar's classic text [22], which is entirely devoted to closedness criteria. For the application to uniform duality, see Duffin, Jeroslow and Karlovitz 13 .

We study the case when the convex set is a cone, using the following framework:

- Given a linear map $M$ between 2 finite dimensional spaces, and its adjoint $M^{*}$,

- a closed, convex cone $K$, and its dual cone $K^{*}=\{y \mid\langle y, x\rangle \geq 0 \forall x \in K\}$,

$(\star)$ When is $M^{*} K^{*}$ closed?

Our main motivation is the following question: is there a common root of the following three well-known, seemingly quite unrelated sufficient conditions?

$$
\begin{aligned}
& \text { ri } K \cap \mathcal{R}(M) \neq \emptyset, \\
& K \cap \mathcal{R}(M)=\text { lspace }(K) \cap \mathcal{R}(M), \\
& K \text { is polyhedral, }
\end{aligned}
$$

where lspace $(K)$ stands for $K \cap(-K)$, the lineality space of $K$.

1.1 A sample of the main results. The paper's main result gives a yes answer, in a surprisingly simple form (see the ensuing explanation for less common notation): 
Theorem 1.1 (Main Theorem) Let $\bar{x} \in \operatorname{ri}(\mathcal{R}(M) \cap K)$, and $F$ the minimal face of $K$ that contains $\bar{x}$. The conditions

(i) $\mathcal{R}(M) \cap \operatorname{dir}(\bar{x}, K)=\mathcal{R}(M) \cap \operatorname{cl} \operatorname{dir}(\bar{x}, K)$;

(ii) $M^{*} F^{\triangle}=M^{*} F^{\perp}$;

(iii) ri $F^{\triangle} \cap \mathcal{N}\left(M^{*}\right) \neq \emptyset$, and $\mathcal{R}(M) \cap F^{\triangle \perp}=\mathcal{R}(M) \cap \operatorname{lin} F$;

(iv) $\mathcal{R}(M) \cap F^{\triangle *}=\mathcal{R}(M) \cap \operatorname{lin} F$;

are equivalent, and necessary for the closedness of $M^{*} K^{*}$. If $K^{*}+F^{\perp}$ is closed, then they are necessary and sufficient.

Here $\operatorname{dir}(\bar{x}, K)=\{y \mid \bar{x}+t y \in K$ for some $t>0\}$ is the set of feasible directions at $\bar{x}$ in $K, F^{\perp}$ is the orthogonal complement of the linear span of $F$,

$$
F^{\triangle}=K^{*} \cap F^{\perp}, F^{\triangle *}=\left(F^{\triangle}\right)^{*}, F^{\triangle \perp}=\left(F^{\triangle}\right)^{\perp} .
$$

It is easy to confirm, why for instance (i) subsumes the three classical conditions:

- if (IMG-RI) holds, then $\bar{x} \in \operatorname{ri} K$, and $\operatorname{dir}(\bar{x}, K)$ is the linear span of $K$, which is a closed set;

- if (IMG-LSPACE $)$ holds, then $\bar{x} \in \operatorname{lspace}(K)$, and $\operatorname{dir}(\bar{x}, K)=K$, which is closed by definition;

- if ( $(\overline{\text { POL }})$ holds, then $\operatorname{dir}(\bar{x}, K)$ is closed, regardless of where $\bar{x}$ is in $K$.

The class of cones, for which the Main Theorem provides a necessary and sufficient condition for an arbitrary $M$, is in fact, quite large.

Definition 1.1 A closed convex cone $C$ is called nice, if

$$
\text { the set } C^{*}+E^{\perp} \text { is closed for all } E \text { faces of } C \text {. }
$$

Polyhedral cones are obviously nice; later on we will show that so are the cone of positive semidefinite matrices, and $p$-cones. The above property of cones is first mentioned in a paper of Borwein and Wolkowicz [1], although they do not use this property to study our main problem.

REMARK 1.1 Condition (ii) has an interesting geometric interpretation. If $K$ is nice, then it implies

$$
\begin{array}{lcc}
M^{*} K^{*} & \subsetneq & \operatorname{cl} M^{*} K^{*} \\
M^{*} F^{\triangle} & \subsetneq & M^{*} F^{\perp}
\end{array}
$$

Also,

$$
M^{*} F^{\triangle} \subseteq M^{*} K^{*}, \text { and } M^{*} F^{\perp} \subseteq \operatorname{cl} M^{*} K^{*},
$$

with the first inclusion being obvious, and the second following from (3.19), shown in the proof of the Main Theorem.

Thus, on the one hand $M^{*} F^{\triangle}$ and $M^{*} F^{\perp}$ act as "substitutes" for $M^{*} K^{*}$ and cl $M^{*} K^{*}$ to check their equality. On the other hand, since $M^{*} F^{\perp}$ is a subspace, the last statement in (1.1) is equivalent to

$$
\operatorname{cl} M^{*} F^{\triangle} \subsetneq M^{*} F^{\perp}
$$

which is the same as

$$
\exists w \in M^{*} F^{\perp} \text { which can be strictly separated from } M^{*} F^{\triangle} \text {. }
$$

We show in Corollary 3.1 that any such $w$ is also in $\mathrm{cl}^{*} K^{*} \backslash M^{*} K^{*}$. However, it provides a stronger certificate of nonclosedness than an arbitrary point in $\operatorname{cl}^{*} K^{*} \backslash M^{*} K^{*}$ : the latter cannot be strictly separated from $M^{*} K^{*}$, while $w$ can be strictly separated from the "substitute" of $M^{*} K^{*}$, namely $M^{*} F^{\triangle}$.

Our problem frequently appears in a different guise: given closed, convex cones $K_{1}$ and $K_{2}$,

$$
(\triangle) \text { When is } K_{1}^{*}+K_{2}^{*} \text { closed? }
$$

A necessary and/or sufficient condition for either one of $(\star)$ and $(\triangle)$ yields such a condition for the other, as explained in Section 5 . 
1.2 Literature review The first reference that we are aware of, which implies the sufficiency of (IMG-RI) is Theorem 2 in Duffin [14. (The proof in Duffin [14 only works in the case when $K$ is fulldimensional - for the general case, one needs to modify it.) The sufficiency of (POL) follows from the fact that a polyhedral cone is finitely generated, so its linear image is also polyhedral. We are not aware of a reference for condition (IMG-LSPACE), so we give a simple proof later on as part of Theorem 2.2 in Section 2

Conditions (IMG-RI), (IMG-LSPACE), and (POL) have their dual counterparts; they are equivalent to

$$
\begin{aligned}
K^{*} \cap \mathcal{N}\left(M^{*}\right) & =K^{\perp} \cap \mathcal{N}\left(M^{*}\right), \\
\text { ri } K^{*} \cap \mathcal{N}\left(M^{*}\right) & \neq \emptyset, \\
K^{*} & \text { is polyhedral, }
\end{aligned}
$$

respectively. The equivalence of (IMG-RI) and (IMG-LSPACE-DUAL) (and of the symmetric pair (IMG-LSPACE) and (IMG-RI-DUAL) will be explained and proved as part of Theorem 2.2 as well.

Theorem 9.1 in Rockafellar 22 implies that for an arbitrary closed convex set $C$, and linear map $A$ the following condition is sufficient for the closedness of $A C$ :

$$
\operatorname{rec}(C) \cap \mathcal{N}(A)=\text { lspace }(\operatorname{rec}(C)) \cap \mathcal{N}(A) .
$$

Here

$$
\operatorname{rec}(C)=\{y \mid x+t y \in C, \forall x \in C, \forall t \geq 0\}
$$

is the recession cone of $C$. This conditon generalizes (IMG-LSPACE-DUAL); it does not seem to have a "primal" counterpart, when $C$ is not a cone. (Theorem 9.1. is in fact more general; it gives a sufficient condition for $\operatorname{cl} A C=A(\operatorname{cl} C)$ to hold, even when $C$ is not closed).

Besides the classical results listed above, several more are available for $(\star)$ and/or $(\triangle)$. We list all that are known to us:

- A sufficient condition for $(\triangle)$ was given by Waksman and Epelman [25, page 95], which for $(\star)$ translates into

$$
\forall y \in \mathcal{N}\left(M^{*}\right) \cap K^{*}: \operatorname{dir}\left(y, K^{*}\right) \text { is closed. }
$$

- Auslender in 2] gave a necessary and sufficient condition for the linear image of an arbitrary closed convex set to be closed.

- Bauschke and Borwein in [7] present a necessary and sufficient condition for the continuous image of a closed convex cone to be closed, in terms of the strong conical hull intersection property.

- Ramana's extended dual in [20 has the following connection to our work: when $K=K^{*}$ is the cone of positive semidefinite matrices, and $b$ a given vector, then his results imply: we can check $b \notin M^{*} K^{*}$ by verifying the feasibility of a semidefinite system, whose size is polynomial in terms of the original data.

Of these four results, the one closest to ours in spirit is the provision (WE); it is an elegant weakening of (IMG-LSPACE-DUAL) and (POL-DUAL). However - in contrast with our conditions - no interesting class of cones has been identified, for which (WE) would be necessary and sufficient. For many relevant cones, such as the semidefinite and second order cones, (WE) reduces to (IMG-LSPACE-DUAL), or a restricted version of (IMG-RI-DUAL): we show this in Section 5 The results of Auslender and of Bauschke and Borwein are more general than ours; however, their conditions on closedness are also more involved.

The rest of the article is structured as follows. Section 2 deals with notation, and surveys the necessary, mostly known results to be used later on. For the sake of better insight, we provide some proofs here. Section 3 presents the main results on problem $(\star)$, and shows how from a "certificate" of nonclosedness of $M^{*} K^{*}$ one can actually produce a vector in $\mathrm{cl}^{*} K^{*} \backslash M^{*} K^{*}$. Section 4 gives a variety of examples, and discusses some of the complexity implications of the Main Theorem: we prove that closedness of the linear image of the semidefinite cone can be verified in polynomial time in the real number model of computing. Section 5 contains our results on $(\triangle)$. Lastly, Appendix A furnishes several, more complicated examples on the use of the Main Theorem. 


\section{Preliminaries and notation}

2.1 The frontier of a set We call the difference between the closure of a set $S$, and $S$ the frontier of $S$, and write

$$
\operatorname{fr}(S)=\operatorname{cl} S \backslash S
$$

2.2 Operators, matrices and inner products Linear operators are denoted by capital letters; when a matrix is considered to be an element of a Euclidean space, and not a linear operator, it is usually denoted by a small letter. We denote by $e_{i, n}$ the $i^{t h}$ unit vector in $\mathbb{R}^{n}$; we write $e_{i}$, if the dimension of the space is clear from the context. The vector of all ones in $\mathbb{R}^{n}$ is denoted by $e$; the dimension should be clear from the context. For a vector $x$, and integers $k, \ell$ with $1<k<\ell$ we write $x_{k: \ell}$ for the subvector $\left(x_{k}, \ldots, x_{\ell}\right)^{T}$.

The range space of an operator $A$ [of a matrix $x]$ is denoted by $\mathcal{R}(A)[\mathcal{R}(x)]$. The orthogonal projection operator onto a linear space $L$ is denoted by $\operatorname{Proj}_{L}()$.

If $S$ is a set, then its linear span is denoted by $\operatorname{lin} S$, and the orthogonal complement of lin $S$ by $S^{\perp}$. For a vector $\bar{x}$, we denote by $\mathbb{R} \bar{x}, \mathbb{R}_{+} \bar{x}$, and $\mathbb{R}_{++} \bar{x}$ the set of all multiples, nonnegative multiples, and strictly positive multiples of $\bar{x}$, respectively.

The inner product of two vectors $x_{1}$ and $x_{2}$ in a Euclidean space is denoted by $\left\langle x_{1}, x_{2}\right\rangle$. Even if the inner products in two different spaces are different, we still use the notation $\langle$,$\rangle for both; ambiguity will$ be prevented by the context.

2.3 A Theorem of Abrams We will extensively use the following

Theorem 2.1 (R. A. Abrams) Let $S$ be an arbitrary set, and $A$ a surjective linear map. Then

(i) $A S$ is closed $\Leftrightarrow S+\mathcal{N}(A)$ is closed.

(ii) $A S$ is not closed, with $A x \in \operatorname{fr}(A S)$, iff $S+\mathcal{N}(A)$ is not closed, with $x \in \operatorname{fr}(S+\mathcal{N}(A))$.

For a proof, see e.g. Berman [8, Lemma 3.1], or Holmes [16, Lemma 17H].

2.4 Cones, faces and complementary faces We assume familiarity with the notions of faces and exposed faces of convex sets; for references see Rockafellar [22, Hiriart-Urruty and Lemarechal [15], or Brondsted [12. If $C$ is a convex set, and $x \in C$, the minimal face of $C$ that contains $x$ is denoted by face $(x, C)$. To denote that $E$ is a face of $C$, we write $E \unlhd C$, and we use the shorthand $E \triangleleft C$ for $E \unlhd C, E \neq C$.

A convex set $C$ is a cone, if $\mu C \subseteq C$ holds for all $\mu \geq 0$. The lineality space of $C$ is defined as

$$
\text { lspace }(C)=C \cap(-C),
$$

and we say that $C$ is pointed, if $\operatorname{lspace}(C)=\{0\}$.

The dual of the convex cone $C$ is

$$
C^{*}=\{z \mid\langle z, x\rangle \geq 0 \text { for all } x \in C\} .
$$

If $C, C_{1}$ and $C_{2}$ are convex cones, then

$$
\begin{aligned}
C^{* *} & =\operatorname{cl} C, \\
\left(C_{1}+C_{2}\right)^{*} & =C_{1}^{*} \cap C_{2}^{*}, \\
\left(C_{1} \cap C_{2}\right)^{*} & =\operatorname{cl}\left(C_{1}^{*}+C_{2}^{*}\right) .
\end{aligned}
$$

Let $E \unlhd C$, and $\bar{x} \in$ ri $E$. Then it is straightforward to see that

$$
C^{*} \cap E^{\perp}=C^{*} \cap\{\bar{x}\}^{\perp} .
$$

The set in 2.7) is denoted by $E^{\triangle}$, and called the complementary (or conjugate) face of $E$. The complementary face of $H \unlhd C^{*}$ is defined as $C \cap H^{\perp}$, and is denoted by $H^{\triangle}$. The reader is warned at this point that the notation ()$^{\triangle}$ is ambiguous, as it uses the same symbol for two different operations: one maps from the faces of $C$ to the faces of $C^{*}$, and one in the other direction. 
The face $\left(E^{\triangle}\right)^{\triangle}$ is the smallest exposed face of $C$ that contains $E$, i.e. the smallest face of $C$ that arises as the intersection of $C$ with a supporting hyperplane, and contains $E$.

The cone $C$ is called facially exposed, if all of its faces are exposed, i.e. they arise as the intersection of $C$ with a supporting hyperplane, in other words, if for all $E \unlhd C,\left(E^{\triangle}\right)^{\triangle}=E$. We remark that it is possible that $C$ is facially exposed, while $C^{*}$ is not.

For brevity, we write $E^{\triangle \triangle}$ for $\left(E^{\triangle}\right)^{\triangle}, E^{\triangle *}$ for $\left(E^{\triangle}\right)^{*}$, and $E^{\triangle \perp}$ for $\left(E^{\triangle}\right)^{\perp}$, if $E \unlhd C$. Some references on the facial structure of convex cones are articles by Barker: [3], 4], [5], and Tam [24].

Definition 2.1 Let $C$ be a closed convex cone. We say that $C$ is nice, if

$$
C^{*}+E^{\perp} \text { is closed } \forall E \unlhd C .
$$

Proposition 2.1 The cone $C$ is nice, if and only if one of the two following statements hold:

$$
\begin{array}{r}
E^{*}=C^{*}+E^{\perp} \forall E \unlhd C, \\
\operatorname{Proj}_{\text {lin } E}\left(C^{*}\right) \quad \text { is closed } \forall E \unlhd C .
\end{array}
$$

Proof. $\quad 2.8) \Leftrightarrow 2.9)$ : This equivalence follows, since

$$
\begin{aligned}
E & =C \cap \operatorname{lin} E \Rightarrow \\
E^{*} & =\operatorname{cl}\left(C^{*}+E^{\perp}\right) \quad(\text { by } 2.6) .
\end{aligned}
$$

$2.8 \Leftrightarrow \Leftrightarrow 2.10$ : We will use Theorem 2.1 with $S=C^{*}$, and $A$ the orthogonal projection operator onto $\operatorname{lin} E$, that is, $A=B\left(B^{*} B\right)^{-1} B^{*}$, where $B$ is any injective linear operator with $\mathcal{R}(B)=\operatorname{lin} E$. Then the equivalence follows, since $E^{\perp}=\mathcal{N}(A)$.

REMARK 2.1 We remark that

- If $K$ is nice, then $K$ must be facially exposed;

- If $K_{1}$ and $K_{2}$ are nice, then so is $K_{1} \cap K_{2}$, but $K_{1}+K_{2}$ may not be nice, even if it is closed;

- The dual of a nice cone may not be nice; it may not even be facially exposed.

These results will be discussed in detail in the forthcoming paper, Pataki [17].

2.5 Spaces and cones of interest The space of $n$ by $n$ symmetric, and the cone of $n$ by $n$ symmetric, positive semidefinite matrices are denoted by $\mathcal{S}^{n}$, and $\mathcal{S}_{+}^{n}$, respectively. If $x$ is positive semidefinite [positive definite], this is also denoted by $x \succeq 0[x \succ 0]$. The space $\mathcal{S}^{n}$ is equipped with the inner product

$$
\langle x, z\rangle:=\sum_{i, j=1}^{n} x_{i j} z_{i j},
$$

and it is a well-known fact, that $\mathcal{S}_{+}^{n}$ is self-dual with respect to it.

The faces of $\mathcal{S}_{+}^{n}$ have an attractive, and simple description. After applying a rotation $q^{T}()$.$q , any face$ can be brought to the form

$$
F=\operatorname{face}\left(\left(\begin{array}{cc}
I_{r} & 0 \\
0 & 0
\end{array}\right) \mid \mathcal{S}_{+}^{n}\right)=\left\{\left(\begin{array}{cc}
x & 0 \\
0 & 0
\end{array}\right) \mid x \in \mathcal{S}_{+}^{r}\right\} .
$$

For a proof, see Barker and Carlson [6], or Pataki [18, Appendix A] for a somewhat simpler one. For a face of this form we will frequently use the shorthand

$$
F=\left(\begin{array}{cc}
\oplus & 0 \\
0 & 0
\end{array}\right), \operatorname{lin} F=\left(\begin{array}{cc}
\times & 0 \\
0 & 0
\end{array}\right), F^{\triangle}=\left(\begin{array}{cc}
0 & 0 \\
0 & \oplus
\end{array}\right), F^{\triangle *}=\left(\begin{array}{cc}
\times & \times \\
\times & \oplus
\end{array}\right),
$$

when the size of the partition is clear from the context. The $\oplus$ sign denotes a positive semidefinite submatrix, and a $\times$ a submatrix with arbitrary elements. We will also use the same shorthand for an element of $F, F^{\triangle}$, etc. as well. 


\begin{tabular}{|c||c|c|}
\hline$K$ & A typical $F$ & $F^{\triangle}$ \\
\hline \hline $\mathbb{R}_{+}^{n}$ & face $\left((e, 0)^{T}, \mathbb{R}_{+}^{n}\right)$ & face $\left((0, e)^{T}, \mathbb{R}_{+}^{n}\right)$ \\
\hline $\mathcal{S}_{+}^{n}$ & face $\left(\left(\begin{array}{cc}I & 0 \\
0 & 0\end{array}\right), \mathcal{S}_{+}^{n}\right)$ & face $\left(\left(\begin{array}{cc}0 & 0 \\
0 & I\end{array}\right), \mathcal{S}_{+}^{n}\right)$ \\
\hline$K_{p, n}$ & $\operatorname{cone}\left\{\left(\|x\|_{p}, x\right)^{T}\right\}$ & $\operatorname{cone}\left\{\left(\|x\|_{q},-x\right)^{T}\right\}$ \\
\hline
\end{tabular}

Table 1: The faces and complementary faces in $\mathbb{R}_{+}^{n}, \mathcal{S}_{+}^{n}$ and $K_{p, n}$

If $1<p<+\infty$, then the $p$-cone in $n$-space is defined as

$$
K_{p, n}=\left\{\left(x_{1}, x_{2: n}\right) \in \mathbb{R}^{1} \times \mathbb{R}^{n-1} \mid x_{1} \geq\left\|x_{2: n}\right\|_{p}\right\} .
$$

We have $K_{p, n}^{*}=K_{q, n}$, where $\frac{1}{p}+\frac{1}{q}=1$. It straightforward to see that $K_{p, n}$ is full dimensional, pointed, and all of its nontrivial faces (i.e. apart from the origin and itself) are of the form

$$
\mathbb{R}_{+} \bar{x} \text { with } \bar{x}_{1}=\left\|\bar{x}_{2: n}\right\|_{p} .
$$

The second order cone, or Lorentz-cone in $n$-space is $K_{2, n}$. Due to its importance we will use another notation for it as well, and write

$$
\mathbb{S O}(n):=K_{2, n}
$$

The cones $\mathcal{S}_{+}^{n}$, and $K_{p, n}$ are facially exposed. They are also nice; the easiest way to prove this is by showing that they satisfy $(2.10)$. In the case of $\mathcal{S}_{+}^{n}$, the projection in question is just a smaller copy of the original cone. In the case of $\mathbb{S O}(n)$ the linear span of any nontrivial face is a line, and all cones contained in a line are closed. (Recall that a nice cone must be facially exposed, as we show in the forthcoming paper, Pataki [17; this article will not rely on this result, however.)

A list of the typical faces of these cones, with the corresponding complementary faces can be found in Table 1 (with the example of the nonnegative orthant being trivial).

2.6 Minimal cones Let $L$ be a subspace, $C$ a closed convex cone, and

$$
\bar{x} \in \operatorname{ri}(L \cap C), \quad E:=\text { face }(\bar{x}, C) .
$$

Then for any $y \in C \cap L$ there is $z \in C \cap L$ with $\bar{x} \in(y, z)$. As a result, $y$ and $z$ are in $E$, so

$$
L \cap C=L \cap E .
$$

Thus, $E$ is the minimal face of $C$, whose intersection with $L$ is the same as that of $C$ itself.

We can also view $E$ as the maximal face of $C$ that contains a vector of $L$ in its relative interior, since it is easy to see that

$$
\text { ri } E_{i} \cap L \neq \emptyset\left(E_{i} \unlhd C, i=1,2\right) \Rightarrow \operatorname{riface}\left(E_{1} \cup E_{2}, C\right) \cap L \neq \emptyset \text {. }
$$

The face $E$ is called the minimal cone of the conic linear system $L \cap C$, and denoted by mincone $(L \cap C)$.

\subsection{The image of a closed convex cone, and a theorem of the alternative}

Lemma 2.1 Let $M$ be a linear map, $K$ a closed convex cone, and $L$ a subspace. Then

$$
\begin{aligned}
M^{-1} K & =\left(M^{*} K^{*}\right)^{*}, \\
\left(M^{-1} K\right)^{*} & =\operatorname{cl}\left(M^{*} K^{*}\right), \\
\text { If ri } K \cap \mathcal{R}(M) & \neq \emptyset, \text { then }\left(M^{-1} K\right)^{*}=M^{*} K^{*}, \\
M^{-1} L & =\left(M^{*} L^{\perp}\right)^{\perp}, \\
\left(M^{-1} L\right)^{\perp} & =M^{*} L^{\perp} .
\end{aligned}
$$


Proof. The equation (C1) follows by

$$
y \in M^{-1} K \Leftrightarrow M y \in K \quad \Leftrightarrow \quad\langle M y, z\rangle=\left\langle y, M^{*} z\right\rangle \geq 0 \forall z \in K^{*} \quad \Leftrightarrow \quad y \in\left(M^{*} K^{*}\right)^{*},
$$

and (C2) by taking duals. The proof of (C3) is more difficult, and it is omitted. In light of (C2), C3 is clearly equivalent to IMG-RI]. The last two equations come from (C1) and $\mathrm{C} 2$, and using $L^{*}=L^{\perp}$.

THEOREM 2.2 Suppose that $L$ is a subspace, and $C$ is a closed, convex cone. Then the following statements are equivalent.

(i) $L \cap$ ri $C \neq \emptyset$.

(ii) $L^{\perp} \cap\left(C^{*} \backslash C^{\perp}\right)=\emptyset$.

(iii) $L+C=L+(-C)=L+\operatorname{lin} C$.

Proof. $-(i) \Leftrightarrow-(i i)$ : Suppose that $L=\mathcal{R}(A)$ with $A$ a linear operator, and fix $c \in$ ri $C$. For a cone $D$, let us write $x \leq_{D} y$ to denote $y-x \in D$. Then $L \cap$ ri $C=\emptyset$ if and only if the value of the conic linear program

$$
\begin{aligned}
\sup & x_{0} \\
\text { st. } & -A x+c x_{0} \quad \leq_{C} \quad 0
\end{aligned}
$$

is zero, which is equivalent to it having a bounded optimal value. But 2.13 is strictly feasible, i.e. there is $x, x_{0}$ such that $A x-c x_{0} \in \operatorname{ri} C$; clearly $x=0, x_{0}=-1$ will do. So its boundedness is equivalent to the dual program being feasible: see e.g. Duffin [14, or Bonnans and Shapiro [10, or Renegar 21 for more recent treatments of the duality theory of conic linear programs. The dual of (2.13) is

$$
\begin{array}{cccc}
\text { inf } & \langle y, 0\rangle & & \\
y & \geq C^{*} & 0 \\
\text { st. } & -A^{*} y & = & 0 \\
& \langle c, y\rangle & = & 1
\end{array}
$$

But 2.7 with $E=C$ implies that for $y \in C^{*}$ the relation $\langle y, c\rangle>0$ holds, iff $y \notin C^{\perp}$. Hence the feasibility of 2.14 is equivalent to the existence of $y \in \mathcal{N}\left(A^{*}\right) \cap\left(C^{*} \backslash C^{\perp}\right)$.

$(i) \Rightarrow($ iii $)$ : It is enough to prove the first equality, since $\operatorname{lin} C=C-C$. Fix $c \in L \cap$ ri $C$, and let $x \in-C, \ell \in L$. Then for a sufficiently large $\lambda>0$ we get

$$
\begin{aligned}
\lambda c+x & \in C \Rightarrow \\
(\lambda c+x)+\ell & \in C+L \Rightarrow \\
x+\ell & \in C+L,
\end{aligned}
$$

with the second implication following from $\lambda c \in L$. Hence $L+(-C) \subseteq L+C$, and the opposite inclusion follows by taking the negative of both sets.

$($ iii $) \Rightarrow(i)$ : Let $x \in \operatorname{ri} C$. Since $-x \in \operatorname{lin} C$, there exist $\ell \in L, c \in C$ such that

$$
-x=\ell+c
$$

hence $x+c=\ell$ is in $L$, and it is trivially in $\operatorname{ri} C$.

REMARK 2.2 The equivalence $(i) \Leftrightarrow(i i)$ in Theorem 2.2 appears quite frequently in the theory of cones, and conic linear programs. The earliest reference we know of is Theorem 3.5 in Berman [8] in the case when $C$ is full-dimensional.

- with $L=\mathcal{N}(A), C=\mathbb{R}_{+}^{n}$, where $A$ is some linear operator, it yields Stiemke's theorem (see Schrijver [23, page 95]):

there is a vector $x$ with $x>0$, and $A x=0$, if and only if $A^{T} y \geq 0$ implies $A^{T} y=0$.

- with $C=K, L=\mathcal{R}(M)$, it proves the equivalence of conditions (IMG-RI) and IMG-LSPACE-DUAL); 
- with $C=K^{*}, L=\mathcal{N}\left(M^{*}\right)$ it proves the equivalence of conditions (IMG-LSPACE) and (IMG-RI-DUAL).

The equivalence $($ i $) \Leftrightarrow($ iii $)$ is elementary, and we have not been able to find a reference even in the LP case. With $C=K^{*}, L=\mathcal{N}\left(M^{*}\right)$ it proves that (IMG-RI-DUAL) is equivalent to $K^{*}+\mathcal{N}\left(M^{*}\right)=$ $\operatorname{lin} K^{*}+\mathcal{N}\left(M^{*}\right)$; so in this case $M^{*} K^{*}=M^{*}\left(\operatorname{lin} K^{*}\right)$, which is a closed set.

Let $A$ be a linear map, and $S, T$ arbitrary sets. Then clearly

$$
\begin{aligned}
A^{-1}(S) \subseteq A^{-1}(T) & \Leftrightarrow \mathcal{R}(A) \cap S \subseteq \mathcal{R}(A) \cap T, \\
A S \subseteq A T & \Leftrightarrow \mathcal{N}(A)+S \subseteq \mathcal{N}(A)+T .
\end{aligned}
$$

3. Main results on the closedness of $\boldsymbol{M}^{*} \boldsymbol{K}^{*}$ Let $M$ be a linear operator, $K$ a closed convex cone, and fix

$$
\bar{x} \in \operatorname{ri}(\mathcal{R}(M) \cap K), F=\operatorname{face}(\bar{x}, K) .
$$

Recall the notation $F^{\triangle}=K^{*} \cap F^{\perp}, F^{\triangle *}=\left(F^{\triangle}\right)^{*}$.

Lemma $3.1 M^{*} K^{*} \cap M^{*} F^{\perp}=M^{*} F^{\triangle}$.

Proof. The inclusion $\supseteq$ is trivial. To see $\subseteq$, let $y \in M^{*} K^{*} \cap M^{*} F^{\perp}$, i.e.

$$
y=M^{*} u=M^{*} v \text {, with } u \in K^{*}, v \in F^{\perp} .
$$

Then

$$
u-v \in \mathcal{N}\left(M^{*}\right) \cap\left(K^{*}+F^{\perp}\right) \subseteq \mathcal{N}\left(M^{*}\right) \cap F^{*}
$$

Then

$$
\begin{array}{cccc}
\langle\bar{x}, u-v\rangle & = & 0 & \Rightarrow \\
u-v & \in & F^{\perp} & \Rightarrow \\
u & \in & F^{\perp} & \Rightarrow \\
u & \in & F^{\triangle} . &
\end{array}
$$

Here the first statement comes from $\bar{x} \in \mathcal{R}(M), u-v \in \mathcal{N}\left(M^{*}\right)$. The first implication follows from invoking 2.7) with $F$ playing the role of both $C$ and $E$, the second from $v \in F^{\perp}$, and the last from using $u \in K^{*}$.

We now prove the Main Theorem: we first restate it for convenience's sake.

THEOREM 1.1 (Main Theorem) Let $\bar{x}$ and $F$ be as in 3.17). The conditions

(i) $\mathcal{R}(M) \cap \operatorname{dir}(\bar{x}, K)=\mathcal{R}(M) \cap \operatorname{cl} \operatorname{dir}(\bar{x}, K)$;

(ii) $M^{*} F^{\triangle}=M^{*} F^{\perp}$;

(iii) ri $F^{\triangle} \cap \mathcal{N}\left(M^{*}\right) \neq \emptyset$, and $\mathcal{R}(M) \cap F^{\triangle \perp}=\mathcal{R}(M) \cap \operatorname{lin} F$;

(iv) $\mathcal{R}(M) \cap F^{\triangle *}=\mathcal{R}(M) \cap \operatorname{lin} F$.

are equivalent, and necessary for the closedness of $M^{*} K^{*}$. If $K^{*}+F^{\perp}$ is closed, then they are necessary and sufficient.

Proof. $\quad M^{*} K^{*}$ closed $\Rightarrow$ (ii), We have

$$
\begin{aligned}
& \left(M^{-1} K\right)^{*}=\operatorname{cl} M^{*} K^{*} \\
& \left(M^{-1} K\right)^{*}=\left(M^{-1} F\right)^{*}=M^{*} F^{*},
\end{aligned}
$$

with the last equality coming from $\mathcal{R}(M) \cap \operatorname{ri} F \neq \emptyset$, and using C3 in Lemma 2.1. Therefore

$$
\operatorname{cl} M^{*} K^{*}=M^{*} F^{*},
$$

and so $M^{*} K^{*}$ is closed, if and only if

$$
M^{*} K^{*}=M^{*} F^{*}
$$


But 3.20 implies

$$
\begin{array}{cccc}
M^{*} K^{*} & \supseteq & M^{*}\left(K^{*}+F^{\perp}\right) & \Leftrightarrow \\
M^{*} K^{*} & \supseteq & M^{*} F^{\perp} & \Leftrightarrow \\
M^{*} K^{*} \cap M^{*} F^{\perp} & \supseteq & M^{*} F^{\perp} & \Leftrightarrow \\
M^{*} F^{\triangle} & \supseteq & M^{*} F^{\perp} & \Leftrightarrow \\
M^{*} F^{\triangle} & = & M^{*} F^{\perp} &
\end{array}
$$

In (3.21) the only nontrivial equivalence is the third, and this follows from Lemma 3.1 .

$M^{*} K^{*}$ closed $\Leftrightarrow$ (ii), when $K^{*}+F^{\perp}$ is closed: In this case 3.20 and the first equation in 3.21 are equivalent.

$($ ii $) \Leftrightarrow($ iii $)$ : First note

$$
\begin{aligned}
& M^{*} F^{\triangle}=M^{*} F^{\perp} \quad \Leftrightarrow \\
& \mathcal{N}\left(M^{*}\right)+F^{\triangle}=\mathcal{N}\left(M^{*}\right)+F^{\perp} \quad \Leftrightarrow \\
& \mathcal{N}\left(M^{*}\right)+F^{\triangle}=\mathcal{N}\left(M^{*}\right)+\operatorname{lin} F^{\triangle} \text { and } \mathcal{N}\left(M^{*}\right)+\operatorname{lin} F^{\triangle}=\mathcal{N}\left(M^{*}\right)+F^{\perp} \Leftrightarrow \\
& \mathcal{N}\left(M^{*}\right) \cap \operatorname{ri} F^{\triangle} \neq \emptyset \quad \text { and } \mathcal{N}\left(M^{*}\right)+\operatorname{lin} F^{\triangle}=\mathcal{N}\left(M^{*}\right)+F^{\perp} \text {. }
\end{aligned}
$$

The first equivalence is from 2.16), and the second from $F^{\triangle} \subseteq \operatorname{lin} F^{\triangle} \subseteq F^{\perp}$. The third follows from the equivalence (i) $\Leftrightarrow$ (iii) in Theorem 2.2 with $L=\mathcal{N}\left(M^{*}\right), C=F^{\triangle}$. By taking orthogonal complements

$$
\mathcal{N}\left(M^{*}\right)+\operatorname{lin} F^{\triangle}=\mathcal{N}\left(M^{*}\right)+F^{\perp} \Leftrightarrow \mathcal{R}(M) \cap F^{\triangle \perp}=\mathcal{R}(M) \cap \operatorname{lin} F .
$$

$-(i i) \Leftrightarrow-(i v)$ : We have

$\begin{array}{cccc}M^{*} F^{\triangle} & \subsetneq & M^{*} F^{\perp} & \Leftrightarrow \\ {\operatorname{cl~} M^{*} F^{\triangle}} & \subsetneq & M^{*} F^{\perp} & \Leftrightarrow \\ \left(\mathrm{cl} M^{*} F^{\triangle}\right)^{*} & \supsetneq & \left(M^{*} F^{\perp}\right)^{*} & \Leftrightarrow \\ \left(M^{*} F^{\triangle}\right)^{*} & \supsetneq & \left(M^{*} F^{\perp}\right)^{*} & \Leftrightarrow \\ M^{-1}\left(F^{\triangle *}\right) & \supsetneq & M^{-1}(\operatorname{lin} F) & \Leftrightarrow \\ \mathcal{R}(M) \cap F^{\triangle *} & \supsetneq & \mathcal{R}(M) \cap \operatorname{lin} F . & \end{array}$

The first equivalence follows from $M^{*} F^{\perp}$ being a subspace, and the second by noting that both cones in the second equation are closed, hence they are equal if and only if their duals are. The third is obvious from the definition of the dual cone, and the fourth is from Lemma 2.1, and noting that the dual of a subspace is its orthogonal complement. The last equivalence is from (2.15).

$(i v) \Leftrightarrow(i)$ : We need the following

Proposition 3.1

$$
\mathcal{R}(M) \cap \operatorname{lin} F=\mathcal{R}(M) \cap(K+\operatorname{lin} F) .
$$

Proof of Proposition 3.1. We only need to show $\supseteq$. Fix $z \in K, f \in \operatorname{lin} F$ such that

$$
z+f \in \mathcal{R}(M) .
$$

We will show $z \in \operatorname{lin} F$. For $\varepsilon>0$, let

$$
x(\varepsilon):=\bar{x}+\varepsilon(z+f)=(\bar{x}+\varepsilon f)+\varepsilon z .
$$

If $\varepsilon$ is sufficiently small, then clearly

$$
\bar{x}+\varepsilon f \in F \Rightarrow x(\varepsilon) \in K \Rightarrow x(\varepsilon) \in F,
$$

with the second implication coming from $x(\varepsilon) \in \mathcal{R}(M)$. Hence $z \in \operatorname{lin} F$, as required.

To complete the proof of $(i v) \Leftrightarrow(i)$ note that by Proposition 3.1 (iv) is equivalent to

$$
\mathcal{R}(M) \cap F^{\triangle *}=\mathcal{R}(M) \cap(K+\operatorname{lin} F) .
$$

But

$$
\begin{aligned}
K+\operatorname{lin} F & =\operatorname{dir}(\bar{x}, K), \\
F^{\triangle *} & =\operatorname{cl} \operatorname{dir}(\bar{x}, K) ;
\end{aligned}
$$

see for instance (3.2.8) and (3.2.10) in Pataki [18]. Plugging these into 3.22 gives (i), as required. 
REMARK 3.1 For better insight it is worthwhile to work out, why the conditions of the Main Theorem are satisfied, when $K$ is the nonnegative orthant. Let us assume that $M$ maps from $\mathbb{R}^{n}$ to $\mathbb{R}^{m}$, and and also denote by $M$ the corresponding matrix. Let $I_{0}$ be a maximal subset of $\{1, \ldots, m\}$ such that

$$
M x \geq 0 \Rightarrow(M x)_{i}=0 \forall i \in I_{0},
$$

and $I_{+}:=\{1, \ldots m\} \backslash I_{0}$. Then $F$, and its related sets are of the form

$$
F=\left(\begin{array}{c}
\oplus \\
0
\end{array}\right), F^{\triangle}=\left(\begin{array}{c}
0 \\
\oplus
\end{array}\right), F^{\triangle *}=\left(\begin{array}{c}
\times \\
\oplus
\end{array}\right), \operatorname{lin} F=\left(\begin{array}{c}
\times \\
0
\end{array}\right), F^{\perp}=\left(\begin{array}{c}
0 \\
\times
\end{array}\right) .
$$

Here $\oplus$ denotes a nonnegative subvector, $\times$ a subvector with arbitrary components, and we assume that the indices in $I_{+}$are numbered continuously starting from 1 . For a vector $y \in \mathbb{R}^{m}$ we will denote the subvector corresponding to $I_{0}$, and $I_{+}$by $y_{0}$, and $y_{+}$, respectively. Also, $M_{0}$ and $M_{+}$will stand for the submatrix of $M$ with rows in $I_{0}$, and $I_{+}$, respectively (naturally, this notation does not carry over for the rest of the paper!). In linear programming terminology, we say that $M_{0} x \geq 0$ is the subsystem of $M x \geq 0$ consisting of all implicit equalities; see e.g. Chapter 8 in Schrijver [23].

To see why condition (iv) is satisfied, we note that

$$
\begin{aligned}
\mathcal{R}(M) \cap F^{\triangle *} & =\left\{y=M x \mid y_{0} \geq 0\right\} \\
\mathcal{R}(M) \cap \operatorname{lin} F & =\left\{y=M x \mid y_{0}=0\right\} .
\end{aligned}
$$

An elementary proof of why these two sets are equal is in Claim (8) on page 100 in Schrijver [23]. In LP terminology, the equality of these two sets expresses the geometrically intuitive fact, that the inequalities in $M_{0} x \geq 0$ already imply that all of them hold as equalities, irrespective of what the inequalities in $M_{+} x \geq 0$ are. Since $K+\operatorname{lin} F$ now equals $F^{\triangle *}$, this argument also illustrates Proposition 3.1

As to condition (ii), we have

$$
\begin{aligned}
M^{*} F^{\triangle} & =\left\{M_{0}^{T} z \mid z \geq 0\right\}, \\
M^{*} F^{\perp} & =\left\{M_{0}^{T} z \mid z \text { free }\right\} .
\end{aligned}
$$

Farkas' lemma for linear inequalities implies that the equality of these two sets is just a restatement of

$$
\left\{x \mid M_{0} x \geq 0\right\}=\left\{x \mid M_{0} x=0\right\} .
$$

In turn, equation (3.26) is the same as $M^{-1}\left(F^{\triangle *}\right)=M^{-1}(\operatorname{lin} F)$; and this last statement is equivalent to $\mathcal{R}(M) \cap F^{\triangle *}=\mathcal{R}(M) \cap \operatorname{lin} F$.

Finally, condition (iii) is satisfied, since the subspaces $\mathcal{R}(M)$ and $\mathcal{N}\left(M^{*}\right)$ contain a strictly complementary pair of nonnegative vectors, and $F^{\triangle \perp}=\operatorname{lin} F$.

Remark 3.2 Suppose that $K^{*}+F^{\perp}$ is not closed for some $F \unlhd K$. In this case there is a map $M$ such that conditions (ii) through (i) in the Main Theorem hold, but $M^{*} K^{*}$ is not closed: such a self-adjoint map is the orthogonal projection onto lin $F$. Then by the equivalence of $(2.9)$ and 2.10$) M^{*} K^{*}$ is not closed, but $\mathcal{R}(M)=\operatorname{lin} F$, hence condition (iv) in the Main Theorem holds.

That is, the conditions of the Main Theorem are sufficient for the closedness of $M^{*} K^{*}$ for all $M$ (with $\bar{x}, F$, etc. defined by the particular $M$ ) if and only if $K$ is nice.

Conditions (i) and (iv) provide a certificate for the nonclosedness of $M^{*} K^{*}$, equivalently of $K^{*}+$ $\mathcal{N}\left(M^{*}\right)$. It is natural to ask, whether from such a certificate we can construct a point in $\operatorname{fr}\left(M^{*} K^{*}\right)$. The answer is yes, as shown by

Corollary 3.1 Let

$$
\begin{aligned}
z & \in \mathcal{R}(M) \cap\left(F^{\triangle *} \backslash \operatorname{lin} F\right) \\
& =\mathcal{R}(M) \cap(\operatorname{cl} \operatorname{dir}(\bar{x}, K) \backslash \operatorname{dir}(\bar{x}, K)),
\end{aligned}
$$

and suppose that $v$ satisfies

$$
v \in F^{\perp},\langle v, z\rangle<0
$$

Then

$$
\begin{aligned}
v & \in \operatorname{fr}\left(K^{*}+\mathcal{N}\left(M^{*}\right)\right) \text { and } \\
M^{*} v & \in \operatorname{fr}\left(M^{*} K^{*}\right)
\end{aligned}
$$


Proof. Writing $z=M y$ with $y \in M^{-1}\left(F^{\triangle *}\right)$, we have

$$
\left\langle M^{*} v, y\right\rangle=\langle v, M y\rangle=\langle v, z\rangle<0 .
$$

Hence

$$
\begin{aligned}
M^{*} v & \in M^{*} F^{\perp} \backslash\left(M^{-1}\left(F^{\triangle *}\right)\right)^{*} \\
& =M^{*} F^{\perp} \backslash \operatorname{cl} M^{*} F^{\triangle} .
\end{aligned}
$$

Therefore

$$
M^{*} v \in M^{*} F^{*}=\operatorname{cl} M^{*} K^{*},
$$

and Lemma 3.1 implies $M^{*} v \notin M^{*} K^{*}$ (for this to hold, already $M^{*} v \in M^{*} F^{\perp} \backslash M^{*} F^{\triangle}$ would be enough). This proves (3.29), and using (ii) in Theorem 2.1 proves (3.28).

Since

$$
y \in M^{-1}\left(F^{\triangle *}\right) \subseteq\left(M^{-1}\left(F^{\triangle *}\right)\right)^{* *}=\left(\operatorname{cl} M^{*} F^{\triangle}\right)^{*},
$$

$y$ is the normal vector of an hyperplane that strictly separates a point of $M^{*} F^{\perp}$, namely $M^{*} v$ from $M^{*} F^{\triangle}$ (equivalently, from $\mathrm{cl} M^{*} F^{\triangle}$ ).

4. Examples and some complexity issues This section gives a variety of examples: in each one, the Main Theorem is used to prove whether or not a set $M^{*} K^{*}$ is closed, with $M$ a linear map, and $K$ a nice cone. More examples are in Appendix A.

In most examples we also provide an ad hoc argument to prove (non)closedness; these will work with $K^{*}+\mathcal{N}\left(M^{*}\right)$ instead, when it is easier to do so (cf. Theorem 2.1).

The examples in this section are quite simple, so in these it is straightforward to conclude the (non)closedness via the $a d$ hoc argument as well. Examples A.1 and A.2 in Appendix A are more intricate (though not large): for these the ad hoc arguments become quite cumbersome, while the proofs based on the Main Theorem remain concise and transparent.

In each example, we will show:

(i) A face $F$ of $K$, identified by a representative $\bar{x} \in \operatorname{ri} F \cap \mathcal{R}(M)$, and

(ii) (a) When the purpose is proving nonclosedness, a vector $z \in \mathcal{R}(M)$.

(b) When the purpose is proving closedness, a vector $\bar{u} \in K^{*} \cap \mathcal{N}\left(M^{*}\right)$.

Then the conditions of the Main Theorem will be employed as follows:

- Condition (iv) to verify the nonclosedness of $M^{*} K^{*}$ : to this end, we must

(i) Verify

$$
F=\operatorname{mincone}(\mathcal{R}(M) \cap K) .
$$

(ii) Verify

$$
z \in \mathcal{R}(M) \cap\left(F^{\triangle *} \backslash \operatorname{lin} F\right) .
$$

- Condition (iii) for checking the closedness of $M^{*} K^{*}$ : to this end one needs to

(i) Verify that $\bar{u} \in \operatorname{ri} F^{\triangle}$.

(ii) If so, then $F=$ face $(\bar{x}, K)$ must be the minimal cone of $\mathcal{R}(M) \cap K$ (so this does not need to be checked separately!). We then need to check

$$
\mathcal{R}(M) \cap F^{\triangle \perp}=\mathcal{R}(M) \cap \operatorname{lin} F .
$$

In the first group of examples $K=K^{*}=\mathcal{S}_{+}^{n}$. In this case $M: \mathbb{R}^{k} \rightarrow \mathcal{S}^{n}$ and $M^{*}: \mathcal{S}^{n} \rightarrow \mathbb{R}^{k}$ are defined via symmetric matrices $m_{1}, \ldots, m_{k}$ as

$$
\begin{aligned}
M(x) & =\sum_{i=1}^{k} x_{i} m_{i},\left(x=\left(x_{1}, \ldots, x_{k}\right)^{T} \in \mathbb{R}^{k}\right) \\
M^{*}(y) & =\left(\left\langle m_{1}, y\right\rangle, \ldots,\left\langle m_{k}, y\right\rangle\right)^{T},\left(y \in \mathcal{S}^{n}\right) .
\end{aligned}
$$


The matrix $\bar{x}$ will always be of the form

$$
\bar{x}=\left(\begin{array}{cc}
I_{r} & 0 \\
0 & 0
\end{array}\right)
$$

In this case, we recall from 2.11 that the relevant sets to prove closedness/nonclosedness are

$$
F=\left(\begin{array}{ll}
\oplus & 0 \\
0 & 0
\end{array}\right), \operatorname{lin} F=\left(\begin{array}{ll}
\times & 0 \\
0 & 0
\end{array}\right), F^{\triangle}=\left(\begin{array}{ll}
0 & 0 \\
0 & \oplus
\end{array}\right), F^{\triangle *}=\left(\begin{array}{cc}
\times & \times \\
\times & \oplus
\end{array}\right) .
$$

In the examples - even in the more involved ones in Appendix A - it will be straightforward to verify 4.30. As to 4.31,

$$
z \in F^{\triangle *} \backslash \operatorname{lin} F \Leftrightarrow z=\left(\begin{array}{cc}
z_{11} & z_{12} \\
z_{12}^{T} & z_{22}
\end{array}\right) \text {, with } z_{22} \succeq 0 \text {, and }\left(z_{12} \neq 0 \text {, or } z_{22} \neq 0\right) \text {, }
$$

so checking this is a straightforward, polynomial time computation. Note that even if the matrices $m_{1}, \ldots, m_{k}$ are rational, it is still possible that $\bar{x}$ has irrational entries, or rational ones with exponentially many digits; for these issues see e.g. the discussion in Ramana [20]. Hence the computation is only guaranteed to be polynomial in the real number model of computing (see Blum et al. [9]), not in the Turing model.

To establish closedness, we need to first verify that for a pair of positive semidefinite matrices $(\bar{x}, \bar{u})$, $\bar{u} \in \operatorname{riface}(\bar{x}, K)^{\triangle}$,.e. they are strictly complementary (see Alizadeh et al. [1], or Pataki [18]). If $\bar{u}$ is of the form

$$
\bar{u}=\left(\begin{array}{cc}
0 & 0 \\
0 & I_{s}
\end{array}\right)
$$

then this task is obvious: we only need to check whether $r+s=n$. Also, condition $\mathcal{R}(M) \cap F^{\triangle \perp}=$ $\mathcal{R}(M) \cap \operatorname{lin} F$ - the equality of two subspaces - can be confirmed by standard linear algebraic techniques.

Clearly, $M^{*} \mathcal{S}_{+}^{n}$ is closed, if and only if $M_{v}^{*} \mathcal{S}_{+}^{n}$ is, if $v$ is an invertible matrix, and $M_{v}$ the operator whose rangespace is generated by $v^{T} m_{1} v, \ldots, v^{T} m_{k} v$. So, even if $\bar{x}$ is not in the form 4.34 , the procedure to verify nonclosedness is only slightly changed: we first have to compute a matrix $v$ whose columns are appropriately scaled eigenvectors of $\bar{x}$, replace $\bar{x}$ by $v^{T} \bar{x} v$, and $M$ by $M_{v}$. If our aim is to check closedness, and $\bar{u}$ is not in the form (4.36), then we will need to compute a matrix $v$ of appropriately scaled shared eigenvectors of $\bar{x}$ and $\bar{u}$, and replace $\bar{x}$ by $v^{T} \bar{x} v, \bar{u}$ by $v^{T} \bar{u} v$, and $M$ by $M_{v}$.

In fact, these arguments prove:

THEOREM 4.1 Given a linear map $M$,

(i) The closedness of $M^{*} \mathcal{S}_{+}^{n}$ can be verified in polynomial time in the real number model of computing.

(ii) Suppose there is an algorithm that for given $\bar{x} \in \mathcal{S}_{+}^{n}$, can verify in polynomial time in the real number model

$$
\bar{x} \in \operatorname{ri}\left(\mathcal{R}(M) \cap \mathcal{S}_{+}^{n}\right) .
$$

Then the nonclosedness of $M^{*} \mathcal{S}_{+}^{n}$ can be verified in polynomial time in the real number model of computing.

In a forthcoming paper we show that indeed there $i s$ an algorithm as required in (ii) of Theorem 4.1 .

It is not known, whether one can actually compute a matrix $\bar{x}$ in $\operatorname{ri}\left(\mathcal{R}(M) \cap \mathcal{S}_{+}^{n}\right)$ efficiently. At any rate, in our examples - several of which, namely the ones in Appendix A, are quite involved - this is easy by inspection, and so is finding the certificate of nonclosedness $z \in \mathcal{R}(M) \cap\left(F^{\triangle *} \backslash \operatorname{lin} F\right)$. Thus, our machinery seems useful even in handcomputations to recognize the closedness or nonclosedness of $M^{*} \mathcal{S}_{+}^{n}$.

In contrast, an ad hoc argument to verify nonclosedness of $M^{*} K^{*}$, or equivalently of $\mathcal{N}\left(M^{*}\right)+K^{*}$ works by

(i) Guessing that some matrix $v$ is in $\operatorname{fr}\left(\mathcal{N}\left(M^{*}\right)+K^{*}\right)$.

(ii) Proving $v \in \operatorname{cl}\left(\mathcal{N}\left(M^{*}\right)+K^{*}\right)$. 
(iii) Proving $v \notin \mathcal{N}\left(M^{*}\right)+K^{*}$.

Even if one correctly guesses a $v$, step (ii) can be troublesome. Also, the obvious proof - an infinite sequence in $\mathcal{N}\left(M^{*}\right)+K^{*}$ that converges to $v$ - is not polynomial time checkable. Constructing the argument in step (iii) is also a matter of luck unless our machinery is used; the same applies to verifying closedness of $M^{*} \bar{K}^{*}$, when it is closed.

EXAmPle 4.1 Let $M: \mathbb{R}^{2} \rightarrow \mathcal{S}^{2}, K=K^{*}=\mathcal{S}_{+}^{2}$,

$$
m_{1}=\left(\begin{array}{ll}
1 & 0 \\
0 & 0
\end{array}\right), m_{2}=\left(\begin{array}{ll}
0 & 1 \\
1 & 0
\end{array}\right), \bar{x}=m_{1} .
$$

Now $M^{*} K^{*}$ is not closed.

- Let us first confirm this by using the Main Theorem. Obviously $F=$ face $(\bar{x}, K)$ equals $\operatorname{mincone}(\mathcal{R}(M) \cap K)$. Since

$$
F=\left(\begin{array}{cc}
\oplus & 0 \\
0 & 0
\end{array}\right), \operatorname{lin} F=\left(\begin{array}{cc}
\times & 0 \\
0 & 0
\end{array}\right), F^{\triangle}=\left(\begin{array}{cc}
0 & 0 \\
0 & \oplus
\end{array}\right), F^{\triangle *}=\left(\begin{array}{cc}
\times & \times \\
\times & \oplus
\end{array}\right),
$$

hence

$$
m_{2} \in \mathcal{R}(M) \cap\left(F^{\triangle *} \backslash \operatorname{lin} F\right),
$$

so the nonclosedness follows from condition (iv). Note that

$$
\bar{u}=\left(\begin{array}{ll}
0 & 0 \\
0 & 1
\end{array}\right) \in \mathcal{N}\left(M^{*}\right) \cap \operatorname{ri~} F^{\triangle},
$$

hence the first part of criterion (iii) does hold.

- Next we produce a vector in $\operatorname{fr}\left(M^{*} K^{*}\right)$ using the recipe of Corollary 3.1. Clearly,

$$
\begin{aligned}
& v \in F^{\perp}, \quad\left\langle v, m_{2}\right\rangle<0 \Leftrightarrow \\
& v_{11}=0, \quad v_{12}<0 .
\end{aligned}
$$

The set of all solutions appropriately normalized is

$$
v=\left(\begin{array}{cc}
0 & -1 \\
-1 & v_{22}
\end{array}\right) \text {, for some } v_{22} \text {. }
$$

Then

$$
w=M^{*} v=(0,-2) \quad \in \quad \operatorname{fr}\left(M^{*} K^{*}\right),
$$

and

$$
v \in \operatorname{fr}\left(K^{*}+\mathcal{N}\left(M^{*}\right)\right) .
$$

- We can prove nonclosedness by verifying (4.39) via an ad hoc argument. For simplicity, assume $v_{22}=0$. Since

$$
\underbrace{\left(\begin{array}{cc}
\varepsilon & -1 \\
-1 & 1 / \varepsilon
\end{array}\right)}_{\in K^{*}}+\underbrace{\left(\begin{array}{cc}
0 & 0 \\
0 & -1 / \varepsilon
\end{array}\right)}_{\in \mathcal{N}\left(M^{*}\right)}=\left(\begin{array}{cc}
\varepsilon & -1 \\
-1 & 0
\end{array}\right) \rightarrow v \text {, as } \varepsilon \searrow 0,
$$

we conclude $v \in \operatorname{cl}\left(\mathcal{N}\left(M^{*}\right)+K^{*}\right)$. But $\mathcal{N}\left(M^{*}\right)$ consists of the multiples of the matrix

$$
p_{1}=\left(\begin{array}{ll}
0 & 0 \\
0 & 1
\end{array}\right) \text {. }
$$

Since we cannot make $v$ positive semidefinite by adding any multiple of $p_{1}$ to it, we obtain $v \notin \mathcal{N}\left(M^{*}\right)+K^{*}$.

- Some remarks on the structure of $M^{*} K^{*}$ :

- in this example $M^{*} F^{\triangle}$ is closed: it is simply $\{(0,0)\}$. 
- It is easy to see that

$$
\operatorname{fr}\left(M^{*} K^{*}\right)=\{(0, \lambda) \mid \lambda \neq 0\},
$$

so all elements of $\operatorname{fr}\left(M^{*} K^{*}\right)$ arise from the recipe of Corollary 3.1. if $z=-m_{2} / 2$, then

$$
v=\left(\begin{array}{ll}
0 & \lambda \\
\lambda & 0
\end{array}\right)
$$

satisfies (3.27), and $M^{*} v=(0, \lambda)$. In particular, $-w=(0,2) \in \operatorname{fr}\left(M^{*} K^{*}\right)$.

EXAMPLE 4.2 Let $M: \mathbb{R}^{2} \rightarrow \mathcal{S}^{3}, K=K^{*}=\mathcal{S}_{+}^{3}$,

$$
m_{1}=\left(\begin{array}{lll}
1 & 0 & 0 \\
0 & 0 & 0 \\
0 & 0 & 0
\end{array}\right), m_{2}=\left(\begin{array}{lll}
0 & 0 & 1 \\
0 & 0 & 1 \\
1 & 1 & 0
\end{array}\right), \bar{x}=m_{1}
$$

Now $M^{*} K^{*}$ is closed, although neither one of the classical conditions (IMG-RI), or (IMG-LSPACE) hold. To see this

- using criterion (iii) in the Main Theorem, note that

$$
\bar{x}=\left(\begin{array}{lll}
1 & 0 & 0 \\
0 & 0 & 0 \\
0 & 0 & 0
\end{array}\right) \in K \cap \mathcal{R}(M), \bar{u}=\left(\begin{array}{lll}
0 & 0 & 0 \\
0 & 1 & 0 \\
0 & 0 & 1
\end{array}\right) \in K^{*} \cap \mathcal{N}\left(M^{*}\right)
$$

are a strictly complementary pair, so $F=\operatorname{face}(\bar{x}, K)$ is the minimal cone of $K \cap \mathcal{R}(M)$. Therefore, $F$ and its related sets look like

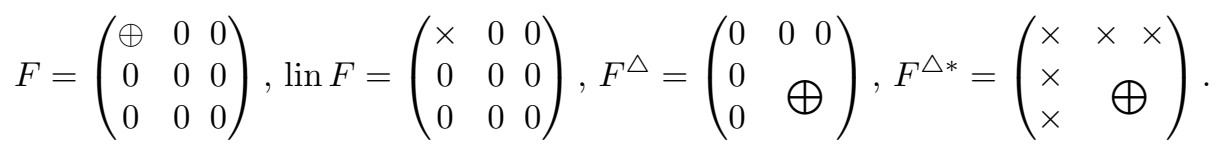

The second part of condition (iii) is straightforward to check.

- directly, observe

$$
M^{*} K^{*}=\mathbb{R}_{+} \times \mathbb{R}
$$

Next we give an example with the second order cone. Now $M: \mathbb{R}^{k} \rightarrow \mathbb{R}^{n}$ and $M^{*}: \mathbb{R}^{n} \rightarrow \mathbb{R}^{k}$ are defined via vectors $m_{1}, \ldots, m_{k}$ as

$$
\begin{aligned}
M(x) & =\sum_{i=1}^{k} x_{i} m_{i}\left(x=\left(x_{1}, \ldots, x_{m}\right)^{T} \in \mathbb{R}^{k}\right) \\
M^{*}(y) & =\left(\left\langle m_{1}, y\right\rangle, \ldots,\left\langle m_{k}, y\right\rangle\right)^{T}\left(y \in \mathbb{R}^{n}\right) .
\end{aligned}
$$

EXAMPLE 4.3 Let $M: \mathbb{R}^{2} \rightarrow \mathbb{R}^{3}, K=K^{*}=\mathbb{S O}(3)$,

$$
m_{1}=\left(\begin{array}{l}
1 \\
1 \\
0
\end{array}\right), m_{2}=\left(\begin{array}{l}
0 \\
0 \\
1
\end{array}\right), \bar{x}=m_{1}
$$

Now $M^{*} K^{*}$ is not closed.

- We can check the nonclosedness of $M^{*} K^{*}$ by using condition (iv) in the Main Theorem: since $F=\operatorname{face}(\bar{x}, K)$ is again trivially the minimal cone of $\mathcal{R}(M) \cap \bar{K}$, so

$$
\operatorname{lin} F=\mathbb{R}\left(\begin{array}{l}
1 \\
1 \\
0
\end{array}\right), F^{\triangle}=\mathbb{R}_{+}\left(\begin{array}{r}
1 \\
-1 \\
0
\end{array}\right)
$$

and therefore

$$
m_{2} \in \mathcal{R}(M) \cap\left(F^{\triangle *} \backslash \operatorname{lin} F\right)
$$

proves nonclosedness. 
- We now find a vector in $\operatorname{fr}\left(M^{*} K^{*}\right)$ via our recipe:

$$
v \in F^{\perp} \Leftrightarrow v=\left(\begin{array}{c}
v_{1} \\
-v_{1} \\
v_{3}
\end{array}\right) \text { for some } v_{1}, v_{3}
$$

so $v$ is a solution of (3.27) with $z=m_{2}$ iff $v_{3}<0$. So

$$
v=\left(\begin{array}{c}
v_{1} \\
-v_{1} \\
-1
\end{array}\right) \in \operatorname{fr}\left(K^{*}+\mathcal{N}\left(M^{*}\right)\right), M^{*} v=(0,-1)^{T} \in \operatorname{fr}\left(M^{*} K^{*}\right)
$$

for any $v_{1}$.

- To check the nonclosedness by an ad hoc argument, we will prove

$$
v=\left(\begin{array}{c}
0 \\
0 \\
-1
\end{array}\right) \in \operatorname{fr}\left(K^{*}+\mathcal{N}\left(M^{*}\right)\right) \text {. }
$$

First note that $\mathcal{N}\left(M^{*}\right)$ consists of the multiples of the vector

$$
p=\left(\begin{array}{c}
1 \\
-1 \\
0
\end{array}\right) \text {. }
$$

Therefore, $v \in \operatorname{cl}\left(K^{*}+\mathcal{N}\left(M^{*}\right)\right)$ follows, if for some suitable $\mu$

$$
v(\varepsilon, \mu)=\left(\begin{array}{c}
\mu \\
-\mu+\varepsilon \\
-1
\end{array}\right) \in \mathbb{S O}(3), \text { as } \varepsilon \searrow 0,
$$

since $\|v(\varepsilon, \mu)-\mu p-v\|=\varepsilon$. But a simple calculation shows that

$$
\mu \geq \frac{\varepsilon}{2}+\frac{1}{2 \varepsilon}
$$

satisfies 4.43).

We cannot make $v$ belong to $\mathbb{S O}(3)$ by adding any multiple of $p$ to it; as a result, $v \notin K^{*}+\mathcal{N}\left(M^{*}\right)$.

5. On the closedness of the sum of two closed cones In this section we study the relationship of the two problems that we recall from Section 1 .

Given a closed, convex cone $K$, its dual cone $K^{*}$, and a linear map $M$,

$(\star)$ When is $M^{*} K^{*}$ closed?

Given closed, convex cones $K_{1}$ and $K_{2}$,

$(\triangle)$ When is $K_{1}^{*}+K_{2}^{*}$ closed?

The two are equivalent in the sense that a necessary and/or sufficient condition for either one yields such a condition for the other:

We can apply a condition for $(\star)$ to derive one for $(\triangle)$ : take

$$
K=K_{1} \times K_{2}, K^{*}=K_{1}^{*} \times K_{2}^{*}, M(x)=(x, x), M^{*}\left(y_{1}, y_{2}\right)=y_{1}+y_{2} .
$$

This way, (IMG-RI), IMG-LSPACE), IMG-LSPACE-DUAL) and (IMG-RI-DUAL respectively yield the sufficient conditions

$$
\begin{aligned}
\text { ri } K_{1} \cap \operatorname{ri} K_{2} & \neq \emptyset, \\
K_{1} \cap K_{2} & =1 \operatorname{space}\left(K_{1}\right) \cap \operatorname{lspace}\left(K_{2}\right), \\
K_{1}^{*} \cap\left(-K_{2}^{*}\right) & =K_{1}^{\perp} \cap K_{2}^{\perp}, \\
\text { ri } K_{1}^{*} \cap\left(-\operatorname{ri} K_{2}^{*}\right) & \neq \emptyset .
\end{aligned}
$$


The applicability of $(\triangle)$ to $(\star)$ seems less well known. Theorem 2.1 implies

$$
M^{*} K^{*} \text { is closed } \Leftrightarrow K^{*}+\mathcal{N}\left(M^{*}\right) \text { is closed. }
$$

Therefore a condition for $(\triangle)$ provides one for $(\star)$ by letting $K_{1}=K, K_{2}=\mathcal{R}(M)$.

A sufficient condition for $(\triangle)$ was given by Waksman and Epelman [25, page 95]. It reads:

$$
\forall x \in K_{1}^{*} \cap\left(-K_{2}^{*}\right): \operatorname{dir}\left(x, K_{1}^{*}\right) \text { and } \operatorname{dir}\left(-x, K_{2}^{*}\right) \text { are closed. }
$$

For $(\star)$ this translates into:

$$
\forall y \in K^{*} \cap \mathcal{N}\left(M^{*}\right): \operatorname{dir}\left(y, K^{*}\right) \text { is closed. }
$$

For many interesting cones, for instance the semidefinite cone, $\operatorname{dir}\left(y, K^{*}\right)$ is closed, only if $y \in \operatorname{ri} K^{*}$, or $y \in K^{\perp}$; see e.g. Ramana et al [19]. The following result shows that for such cones (WE) reduces to the classic condition (IMG-LSPACE-DUAL, or a restricted version of (IMG-RI-DUAL):

Proposition 5.1 Suppose that WE is satisfied by $M^{*}$ and $K^{*}$, and $K^{*}$ is such that: for $y \in K^{*}$, the set $\operatorname{dir}\left(y, K^{*}\right)$ is closed only if $y \in \operatorname{ri} K^{*}$, or $y \in K^{\perp}$. Then

(i) $K^{*} \cap \mathcal{N}\left(M^{*}\right)=K^{\perp} \cap \mathcal{N}\left(M^{*}\right)$, or

(ii) $K^{*} \cap \mathcal{N}\left(M^{*}\right)=\operatorname{cone}\{\bar{y}\}$ for some $\bar{y} \in \operatorname{ri} K^{*}$.

Proof. Let $\bar{y} \in \operatorname{ri}\left(K^{*} \cap \mathcal{N}\left(M^{*}\right)\right)$. Since $\mathrm{WE}$ holds, either $\bar{y} \in K^{\perp}$, or $\bar{y} \in$ ri $K^{*}$. We only need to look at the second case further. Let $z \in K^{*} \cap \mathcal{N}\left(M^{*}\right), z \neq \bar{y}$. If $z \notin$ ri $K^{*}$, then a point on the open line-segment $(z, \bar{y})$ will be in the relative interior of a face distinct from $K^{\perp}$, and $K^{*}$, as the relative interiors of the faces of $K^{*}$ form a partition of $K^{*}$, cf. Rockafellar [22, Theorem 18.2]. Now we only have to exclude

$$
z \in \operatorname{ri} K^{*} \text {, and } z \notin \operatorname{cone}\{\bar{y}\} \text {. }
$$

Suppose to the contrary that 5.46 holds. Let us extend the line segment from $z$ to $\bar{y}$ past $\bar{y}$ [past $z$ ] in ri $K^{*}$, and denote by $u_{1}\left[u_{2}\right]$ the intersection point with the relative boundary of $K^{*}$ (i.e. with $K^{*} \backslash$ ri $K^{*}$ ). At least one of $u_{1}$ and $u_{2}$ is not in $K^{\perp}$ (both being in $K^{\perp}$ would imply $y \in K^{\perp}$ ); suppose this point is $u_{1}$. Then $u_{1} \in K^{*} \cap \mathcal{N}\left(M^{*}\right)$, and $\operatorname{dir}\left(u_{1}, K^{*}\right)$ is not closed, a contradiction.

Our main result follows: the reader can easily check why its conditions follow from (SUM-RI, SUM-LSPACE) and the polyhedrality of $K_{1}$ and $K_{2}$.

Theorem 5.1 (Main Theorem for Sum) Let $\tilde{x} \in \operatorname{ri}\left(K_{1} \cap K_{2}\right), F_{1}=$ face $\left(\tilde{x}, K_{1}\right), F_{2}=$ face $\left(\tilde{x}, K_{2}\right)$. The conditions

(i) $\operatorname{dir}\left(\tilde{x}, K_{1}\right) \cap \operatorname{dir}\left(\tilde{x}, K_{2}\right)=\operatorname{cl} \operatorname{dir}\left(\tilde{x}, K_{1}\right) \cap \operatorname{cl} \operatorname{dir}\left(\tilde{x}, K_{2}\right)$.

(ii) $F_{1}^{\triangle}+F_{2}^{\triangle}=F_{1}^{\perp}+F_{2}^{\perp}$.

(iii) ri $F_{1}^{\triangle} \cap-\operatorname{ri} F_{2}^{\triangle} \neq \emptyset$, and $F_{1}^{\triangle \perp} \cap F_{2}^{\triangle \perp}=\operatorname{lin} F_{1} \cap \operatorname{lin} F_{2}$.

(iv) $F_{1}^{\Delta *} \cap F_{2}^{\Delta *}=\operatorname{lin} F_{1} \cap \operatorname{lin} F_{2}$.

are equivalent, and necessary for the closedness of $K_{1}^{*}+K_{2}^{*}$. If $K_{1}^{*}+F_{1}^{\perp}$ and $K_{2}^{*}+F_{2}^{\perp}$ are closed - in particular, if $K_{1}$ and $K_{2}$ are both nice - then they are necessary and sufficient.

Proof. We use the Main Theorem with the choice of $M$ and $K$ prescribed in (5.44). This way

- $\bar{x} \in \operatorname{ri}(\mathcal{R}(M) \cap K) \Leftrightarrow \bar{x}=(\tilde{x}, \tilde{x})$, with $\tilde{x} \in \operatorname{ri}\left(K_{1} \cap K_{2}\right)$.

- $\bar{x} \in \mathcal{R}(M) \cap \operatorname{ri} F$ with $F \unlhd K \Leftrightarrow \tilde{x} \in \operatorname{ri} F_{1} \cap$ ri $F_{2}$ with $F_{1} \unlhd K_{1}, F_{2} \unlhd K_{2}$.

- $\tilde{u} \in \mathcal{N}\left(M^{*}\right) \cap \operatorname{ri} F^{\triangle}$ with $F=F_{1} \times F_{2}, F_{1} \unlhd K_{1}, F_{2} \unlhd K_{2} \Leftrightarrow \tilde{u} \in \operatorname{ri} F_{1}^{\triangle} \cap-\operatorname{ri} F_{2}^{\triangle}$.

Using these correspondences, the conditions of the Main Theorem are equivalent to their counterparts in this theorem. 
REMark 5.1 Following the recipe of Corollary 3.1, if Condition (iv) in the Main Theorem for Sum is violated, then from a given

$$
\begin{aligned}
z & \in\left(F_{1}^{\triangle *} \cap F_{2}^{\triangle *}\right) \backslash\left(\operatorname{lin} F_{1} \cap \operatorname{lin} F_{2}\right) \\
& =\left(\operatorname{cl} \operatorname{dir}\left(\tilde{x}, K_{1}\right) \cap \operatorname{cl} \operatorname{dir}\left(\tilde{x}, K_{2}\right)\right) \backslash\left(\operatorname{dir}\left(\tilde{x}, K_{1}\right) \cap \operatorname{dir}\left(\tilde{x}, K_{2}\right)\right)
\end{aligned}
$$

we can construct

$$
w \in\left(F_{1}^{\perp}+F_{2}^{\perp}\right) \backslash \operatorname{cl}\left(F_{1}^{\triangle}+F_{2}^{\triangle}\right) \subseteq \operatorname{fr}\left(K_{1}^{*}+K_{2}^{*}\right),
$$

as follows (we leave working out the exact correspondence to the reader): We find $\left(v_{1}, v_{2}\right)$ satisfying

$$
\left(v_{1}, v_{2}\right) \in F_{1}^{\perp} \times F_{2}^{\perp},\left\langle v_{1}+v_{2}, z\right\rangle<0,
$$

then take $w=v_{1}+v_{2}$.

In fact, the system (5.47) has a solution, iff it has one with $v_{1}=0$, or one with $v_{2}=0$. The reason is as follows: Condition (ii) in the Main Theorem for Sum is violated, if and only if

$$
\begin{array}{lcl}
\operatorname{cl}\left(F_{1}^{\triangle}+F_{2}^{\triangle}\right) & \subsetneq F_{1}^{\perp}+F_{2}^{\perp} \Leftrightarrow \\
\operatorname{cl}\left(F_{1}^{\triangle}+F_{2}^{\triangle}\right) & \subsetneq & F_{1}^{\perp} \text { or } \operatorname{cl}\left(F_{1}^{\triangle}+F_{2}^{\triangle}\right) \subsetneq F_{2}^{\perp} .
\end{array}
$$

If the first case in $(5.49)$ holds, and $v_{1}$ is in the difference of the corresponding sets, then $\left(v_{1}, 0\right)$ satisfies 5.47); if the second case in (5.49) holds, and $v_{2}$ is in the difference of the sets, then $\left(0, v_{2}\right)$ satisfies (5.47).

EXAMPLE 5.1 Let

then

$$
K_{1}=\mathcal{S}_{+}^{2}, K_{2}=\left\{x \in \mathcal{S}^{2} \mid\left\langle x,\left(\begin{array}{ll}
0 & 0 \\
0 & 1
\end{array}\right)\right\rangle \leq 0\right\},
$$

$$
K_{1}^{*}=\mathcal{S}_{+}^{2}, K_{2}^{*}=\operatorname{cone}\left\{\left(\begin{array}{cc}
0 & 0 \\
0 & -1
\end{array}\right)\right\} .
$$

Using the notation of the Main Theorem for Sum,

$$
\tilde{x}=\left(\begin{array}{ll}
1 & 0 \\
0 & 0
\end{array}\right), F_{1}=\left(\begin{array}{cc}
\oplus & 0 \\
0 & 0
\end{array}\right), F_{2}=\left\{x \in \mathcal{S}^{2} \mid\left\langle x,\left(\begin{array}{cc}
0 & 0 \\
0 & 1
\end{array}\right)\right\rangle=0\right\},
$$

hence

$$
F_{1}^{\triangle *}=\left(\begin{array}{cc}
\times & \times \\
\times & \oplus
\end{array}\right), F_{2}^{\triangle}=K_{2}^{*}, F_{2}^{\triangle *}=K_{2} .
$$

Since

$$
z=\left(\begin{array}{ll}
0 & 1 \\
1 & 0
\end{array}\right) \in\left(F_{1}^{\triangle *} \cap F_{2}^{\triangle *}\right) \backslash \operatorname{lin} F_{1},
$$

we conclude that $K_{1}^{*}+K_{2}^{*}$ is not closed. Solving (5.47) with $v_{2}=0$ gives

$$
v_{1}=\left(\begin{array}{cc}
0 & -1 \\
-1 & 0
\end{array}\right) \cdot \in F_{1}^{\perp} \backslash \operatorname{cl}\left(F_{1}^{\triangle}+F_{2}^{\triangle}\right) \subseteq \operatorname{fr}\left(K_{1}^{*}+K_{2}^{*}\right) .
$$

The fact that $v_{1}$ is in $\operatorname{fr}\left(K_{1}^{*}+K_{2}^{*}\right)$ is also easy to check directly.

Of course, nonclosedness of $K_{1}^{*}+K_{2}^{*}$ also follows from the fact that it is equal to $K_{1}^{*}+\operatorname{lin} K_{2}^{*}$, and the latter set is the same as $K^{*}+\mathcal{N}\left(M^{*}\right)$ of Example 4.1, where its nonclosedness was already proven.

Appendix A. More examples on the closedness/nonclosedness of $M^{*} K^{*}$ In this appendix we give several, more involved examples of mappings $M: \mathbb{R}^{m} \rightarrow \mathcal{S}^{n}$ for some $m, n$ integers. In these proving closedness or nonclosedess of $M^{*} K^{*}$ will be quite nontrivial via ad hoc arguments, but still straightforward using the conditions of the Main Theorem.

Example A.1 Let $M: \mathbb{R}^{5} \rightarrow \mathcal{S}^{4}, K=K^{*}=\mathcal{S}_{+}^{4}$, and the generators of $\mathcal{R}(M)$ called $m_{1}, \ldots, m_{5}$ as below:

$$
\left(\begin{array}{llll}
1 & 0 & 0 & 0 \\
0 & 0 & 0 & 0 \\
0 & 0 & 0 & 0 \\
0 & 0 & 0 & 0
\end{array}\right),\left(\begin{array}{cccc}
0 & -1 & -1 & 0 \\
-1 & 1 & 0 & 0 \\
-1 & 0 & 0 & 0 \\
0 & 0 & 0 & 0
\end{array}\right),\left(\begin{array}{cccc}
0 & 0 & -1 & 1 \\
0 & 0 & 0 & 1 \\
-1 & 0 & 0 & 0 \\
1 & 1 & 0 & 0
\end{array}\right),\left(\begin{array}{cccc}
0 & 3 & 0 & 0 \\
3 & -1 & 1 & 0 \\
0 & 1 & 0 & 0 \\
0 & 0 & 0 & 0
\end{array}\right),\left(\begin{array}{cccc}
0 & 0 & 0 & 0 \\
0 & 0 & 0 & -1 \\
0 & 0 & 1 & 0 \\
0 & -1 & 0 & 0
\end{array}\right),
$$

and

Again, $M^{*} K^{*}$ is not closed.

$$
\bar{x}=m_{1} .
$$


- To confirm this by using the Main Theorem, we will first verify that $F=$ face $(\bar{x}, K)$ equals $\operatorname{mincone}(\mathcal{R}(M) \cap K)$. Suppose

$$
x=\sum_{i=1}^{5} \mu_{i} m_{i} \succeq 0
$$

Then

$$
x_{44}=0 \Rightarrow x_{., 4}=0 \Rightarrow \mu_{3}=\mu_{5}=0 \Rightarrow x_{33}=0 \Rightarrow x_{., 3}=0 \Rightarrow \mu_{2}=\mu_{4}=0 .
$$

Here $x_{., j}$ denotes the $j$ th column of $x$, the first and fourth implications come from the positive semidefiniteness of $x$, and the others are trivial. This proves that $\bar{x}$ - up to a nonnegative factor - is the only positive semidefinite matrix in $\mathcal{R}(M)$; i.e. face $(\bar{x}, K)=\operatorname{mincone}(\mathcal{R}(M) \cap K)$. Thus

$$
m_{2} \in \mathcal{R}(M) \cap\left(F^{\triangle *} \backslash \operatorname{lin} F\right),
$$

proves nonclosedness via condition[iv)] in the Main Theorem. Two matrices in $\operatorname{fr}\left(K^{*}+\mathcal{N}\left(M^{*}\right)\right)$ that can be produced from $m_{2}$ are

$$
v_{1}=\left(\begin{array}{llll}
0 & 1 & 0 & 0 \\
1 & 0 & 0 & 0 \\
0 & 0 & 0 & 0 \\
0 & 0 & 0 & 0
\end{array}\right), v_{2}=\left(\begin{array}{llll}
0 & 0 & 1 & 0 \\
0 & 0 & 0 & 0 \\
1 & 0 & 0 & 0 \\
0 & 0 & 0 & 0
\end{array}\right) .
$$

- Proving nonclosedness without our machinery is quite troublesome. The generators of $\mathcal{N}\left(M^{*}\right)$ can be chosen as

$$
\left(\begin{array}{llll}
0 & 0 & 0 & 0 \\
0 & 0 & 0 & 0 \\
0 & 0 & 0 & 0 \\
0 & 0 & 0 & 1
\end{array}\right),\left(\begin{array}{cccc}
0 & 0 & 0 & -1 \\
0 & 0 & 0 & 1 \\
0 & 0 & 2 & 0 \\
-1 & 1 & 0 & 0
\end{array}\right),\left(\begin{array}{llll}
0 & 0 & 1 & 1 \\
0 & 2 & 1 & 0 \\
1 & 1 & 0 & 0 \\
1 & 0 & 0 & 0
\end{array}\right),\left(\begin{array}{llll}
0 & 0 & 0 & 0 \\
0 & 0 & 0 & 0 \\
0 & 0 & 0 & 1 \\
0 & 0 & 1 & 0
\end{array}\right),\left(\begin{array}{cccc}
0 & -1 & 1 & 1 \\
-1 & 0 & 3 & 0 \\
1 & 3 & 0 & 0 \\
1 & 0 & 0 & 0
\end{array}\right) .
$$

Let us call these matrices $p_{1}, \ldots, p_{5}$ in the above order.

- First we must guess a matrix

$$
w \in \operatorname{fr}\left(K^{*}+\mathcal{N}\left(M^{*}\right)\right) .
$$

By inspection one may think $\pm v_{1}$ and $\pm v_{2}$ to be in , $\operatorname{fr}\left(K^{*}+\mathcal{N}\left(M^{*}\right)\right)$ as they both "look similar" to the matrix $w$ in Example 4.1, and in that example the $\operatorname{set} \operatorname{fr}\left(K^{*}+\mathcal{N}\left(M^{*}\right)\right)$ is symmetric around the origin. However, not all these will work, since

$$
-v_{2}+p_{1}+p_{2}+p_{3}=\left(\begin{array}{cccc}
0 & 0 & 0 & 0 \\
0 & 2 & 1 & 1 \\
0 & 1 & 2 & 0 \\
0 & 1 & 0 & 1
\end{array}\right) \succeq 0
$$

so $-v_{2} \in K^{*}+\mathcal{N}\left(M^{*}\right)$.

- Proving

is easy. Since

$$
v_{1} \in \operatorname{cl}\left(K^{*}+\mathcal{N}\left(M^{*}\right)\right)
$$

$$
\mathcal{R}(M) \cap K=\left(\begin{array}{llll}
\oplus & 0 & 0 & 0 \\
0 & 0 & 0 & 0 \\
0 & 0 & 0 & 0 \\
0 & 0 & 0 & 0
\end{array}\right)
$$

we obtain

$$
\operatorname{cl}\left(\mathcal{N}\left(M^{*}\right)+K^{*}\right)=(\mathcal{R}(M) \cap K)^{*}=\left(\begin{array}{cccc}
\oplus & \times & \times & \times \\
\times & \times & \times & \times \\
\times & \times & \times & \times \\
\times & \times & \times & \times
\end{array}\right),
$$

so A.50 follows. (We remark that it is so easy to calculate $\operatorname{cl}\left(\mathcal{N}\left(M^{*}\right)+K^{*}\right)$ only because $\mathcal{R}(M) \cap K$ is generated by one matrix, namely $\bar{x}$; in general, it would be trickier to show A.50). 
- Next, we verify

$$
v_{1} \notin K^{*}+\mathcal{N}\left(M^{*}\right)
$$

Assume to the contrary that

$$
v_{1}(\mu):=v_{1}+\sum_{i=1}^{5} \mu_{i} p_{i} \succeq 0 \text { for some } \mu_{1}, \ldots, \mu_{5} .
$$

Let us focus only on a part of $v_{1}(\mu)$, and denote the uninteresting components as well as components determined by symmetry by '*':

$$
v_{1}(\mu):=\left(\begin{array}{cccc}
0 & -\mu_{5}+1 & \mu_{3}+\mu_{5} & * \\
* & 2 \mu_{3} & * & * \\
* & * & * & * \\
* & * & * & *
\end{array}\right) \succeq 0
$$

By positive semidefiniteness, we must have $v_{1}(\mu)_{12}=0$, hence $\mu_{5}=1$. This, together with $v_{1}(\mu)_{13}=0$ implies $\mu_{3}=-1$; but this leads to $v_{1}(\mu)_{22}=-2$, a contradiction.

- In comparison to the method based on the Main Theorem, we see that just proving $v_{1} \notin$ $K^{*}+\mathcal{N}\left(M^{*}\right)$ is as hard as verifying $F=\operatorname{mincone}(\mathcal{R}(M) \cap K)$. However, the rest of the proof via the Main Theorem is routine, whereas in the improvised method the other steps are just as involved, or more so.

EXAmple A.2 Let $M: \mathbb{R}^{4} \rightarrow \mathcal{S}^{4}, K=K^{*}=\mathcal{S}_{+}^{4}$,

$$
m_{1}=\left(\begin{array}{llll}
1 & 0 & 0 & 0 \\
0 & 0 & 0 & 0 \\
0 & 0 & 0 & 0 \\
0 & 0 & 0 & 0
\end{array}\right), m_{2}=\left(\begin{array}{cccc}
0 & 1 & 0 & 0 \\
1 & -1 & 0 & 1 \\
0 & 0 & 1 & 0 \\
0 & 1 & 0 & 0
\end{array}\right), m_{3}=\left(\begin{array}{cccc}
0 & 0 & 1 & 0 \\
0 & 1 & 0 & 0 \\
1 & 0 & -1 & 0 \\
0 & 0 & 0 & 0
\end{array}\right), m_{4}=\left(\begin{array}{cccc}
0 & 0 & 0 & 1 \\
0 & 0 & 1 & 0 \\
0 & 1 & 0 & 0 \\
1 & 0 & 0 & 0
\end{array}\right) .
$$

Condition (iii) in the Main Theorem proves closedness of $M^{*} K^{*}$, since

$$
\bar{x}=\left(\begin{array}{llll}
1 & 0 & 0 & 0 \\
0 & 0 & 0 & 0 \\
0 & 0 & 0 & 0 \\
0 & 0 & 0 & 0
\end{array}\right) \in K \cap \mathcal{R}(M), \bar{u}=\left(\begin{array}{cccc}
0 & 0 & 0 & 0 \\
0 & 1 & 0 & 0 \\
0 & 0 & 1 & 0 \\
0 & 0 & 0 & 1
\end{array}\right) \in K^{*} \cap \mathcal{N}\left(M^{*}\right)
$$

are a strictly complementary pair. Hence $F=$ face $(\bar{x}, K)$ is equal to mincone $(\mathcal{R}(M) \cap K)$, and $\mathcal{R}(M) \cap$ $F^{\triangle \perp}=\mathcal{R}(M) \cap \operatorname{lin} F$ is obvious: a matrix $x=\sum_{i=1}^{4} \mu_{i} m_{i}$ can belong to $F^{\triangle \perp}$ (i.e. have its lower 3 by 3 principal minor zero) only if $\mu_{2}=\mu_{3}=\mu_{4}=0$.

In this example we could not think of any reasonably short ad hoc argument to prove closedness.

Acknowledgments. Thanks are due to Stefan Schmieta for his comments, and to Heinz Bauschke for bringing to my attention reference [16, its relevance in establishing the connection of problems $(\star)$ and $(\triangle)$, and reference 7 . Thanks are also due to Tamás Terlaky for an observation leading to equivalence $(i) \Leftrightarrow($ iii) in Theorem 2.2. I also thank the referees for their careful reading of the paper, and their suggestions.

\section{References}

[1] F. Alizadeh, J-P. Haeberly, and M.L. Overton, Complementarity and nondegeneracy in semidefinite programming, Math. Program. 77 (1997), 111-128.

[2] A. Auslender, Closedness criteria for the image of a closed set by a linear operator, Numer. Funct. Anal. Optim. 17 (1996), 503-515.

[3] G.P. Barker, The lattice of faces of a finite dimensional cone, Linear Algebra Appl. 7 (1973), 71-82.

[4] _ Faces and duality in convex cones, Linear Multilinear Algebra 6 (1977), 161-169.

[5] _ _ Theory of cones, Linear Algebra Appl. 39 (1981), 263-291.

[6] G.P. Barker and D. D. Carlson, Cones of diagonally dominant matrices, Pacific J. Math. 57 (1975), $15-32$. 
[7] H. Bauschke and J. M. Borwein, Conical open mapping theorems and regularity, Proceedings of the Centre for Mathematics and its Applications 36, Australian National University, 1999, pp. 1-10.

[8] A. Berman, Cones, matrices and mathematical programming, Springer, 1973.

[9] L. Blum, F. Cucker, M. Shub, and S. Smale, Complexity and real computation, Springer, 1998.

[10] J.F. Bonnans and A. Shapiro, Perturbation analysis of optimization problems, Springer, 2000.

[11] J.M. Borwein and H. Wolkowicz, Regularizing the abstract convex program, J. Math. Anal. Appl. 83 (1981), 495-530.

[12] A. Brondsted, An introduction to convex polytopes, Springer, 1983.

[13] R. J. Duffin, R. G. Jeroslow, and L. A. Karlovitz, Duality in semi-infinite linear programming, Semiinfinite programming and applications (Austin, Tex., 1981), Lecture Notes in Econom. and Math. Systems, vol. 215, Springer, 1983, pp. 50-62.

[14] R.J. Duffin, Infinite programs, Linear Equalities and Related Systems (A.W. Tucker, ed.), Princeton University Press, Princeton, NJ, 1956, pp. 157-170.

[15] J.B. Hiriart-Urruty and C. Lemarechal, Convex analysis and minimization algorithms, Springer, 1993.

[16] R. B. Holmes, Geometric functional analysis and its applications, Springer, 1975.

[17] G. Pataki, A partial characterization of nice cones, forthcoming.

[18] _ The geometry of semidefinite programming, Handbook of SEMIDEFINITE PROGRAMMING (R. Saigal, L. Vandenberghe, and H. Wolkowicz, eds.), Kluwer Academic Publishers, Waterloo, Canada, 2000.

[19] M. Ramana, L. Tuncel, and H. Wolkowicz, Strong duality for semidefinite programming, SIAM J. Optim 7 (1997), no. 3, 641-662.

[20] M. V. Ramana, An exact duality theory for semidefinite programming and its complexity implications, Math. Program. 77 (1997), 129-162.

[21] J. Renegar, A mathematical view of interior-point methods in convex optimization, MPS-SIAM Series on Optimization, SIAM, Philadelphia, USA, 2001.

[22] R.T. Rockafellar, Convex analysis, Princeton University Press, Princeton, NJ, USA, 1970.

[23] A. Schrijver, Theory of linear and integer programming, John Wiley \& Sons, New York, 1986.

[24] B.-S. Tam, On the duality operator of a convex cone, Linear Algebra Appl. 64 (1985), 33-56.

[25] Z. Waksman and M. Epelman, On point classification in convex sets, Math. Scand. 38 (1976), 83-96. 